\title{
The relationship between post-onset pregnancy and functional outcome in women with recent onset inflammatory polyarthritis: results from the Norfolk Arthritis Register
}

\author{
E M Camacho, ${ }^{1}$ T M Farragher, ${ }^{1,2}$ M Lunt, ${ }^{1}$ S M M Verstappen, ${ }^{1}$ D Bunn, ${ }^{1,3}$ \\ D P M Symmons ${ }^{1}$
}

- Additional data are published online only. To view these files please visit the journal online (http://ard.bmj.com).

${ }^{1}$ Arthritis Research UK Epidemiology Unit, The University of Manchester, Manchester, UK

${ }^{2}$ Centre for Medical Statistics and Health Evaluation, University of Liverpool, Liverpool UK

${ }^{3}$ Department of Rheumatology, Norfolk and Norwich University Hospital, Norwich, UK

\section{Correspondence to} D P M Symmons, Arthritis Research UK Epidemiology Unit, Manchester Academic Health Sciences Centre, University of Manchester, Stopford Building, Oxford Road, Manchester M13 9PT, UK;

deborah.symmons@

manchester.ac.uk

Accepted 13 May 2010

\section{ABSTRACT}

Objective To examine the influence of post-symptomonset pregnancy on disease outcome in women with inflammatory polyarthritis (IP).

Methods A total of 631 women, aged $<48$ years at symptom onset, were registered with the Norfolk Arthritis Register (NOAR) between 1990 and 2004. Functional disability was assessed using the Stanford Health Assessment Questionnaire (HAO). Blood was tested for rheumatoid factor (RF) and anti-cyclic citrullinated peptide antibody (ACPA). The date and outcome of all pregnancies were reported during a median follow-up of 7 years. Linear random effects models were used to examine HAO score over time, by pregnancy status. Results were then stratified for RF and ACPA status. Results In all, 72 women had a post-onset pregnancy (Po-P) including 45 women who were pregnant at a follow-up assessment. Pregnancy was generally associated with lower HAO scores over time than nonpregnancy. The 10 ACPA-positive women who had a Po-P had significantly worse subsequent $\mathrm{HAO}$ scores.

Conclusion Overall, Po-P is associated with lower $\mathrm{HAO}$ scores, compared to no Po-P. This may reflect a beneficial effect of pregnancy on disease outcome, or that predominantly women with milder disease become pregnant. In women with the worst predicted outcome (APCA positive), Po-P is associated with a worse outcome than no pregnancy.

Hench first reported the association between pregnancy and an improvement in symptoms of inflammatory polyarthritis (IP) and rheumatoid arthritis (RA) in 1935. ${ }^{1}$ Since then a number of studies have replicated the observation ${ }^{2}{ }^{3}$ and results from the Physical Activity in Rheumatoid Arthritis (PARA) study suggest that rheumatoid factor (RF) and anti-cyclic citrullinated peptide antibody (ACPA)negative women are more likely to show an improvement of RA symptoms during pregnancy than autoantibody-positive women. ${ }^{4}$

The long-term effects of pregnancy on RA outcome have been less studied. A retrospective study of 200 Finnish women patients with RA, half of whom had a post-onset pregnancy (Po-P), reported no significant effect of Po-P on prognosis. ${ }^{5}$ A prospectively followed inception cohort of 132 Dutch women with RA, 24 of whom had a Po-P reported no significant effect of Po-P on cross-sectional disease outcome at 12 years. ${ }^{6}$ We sought to contribute to this area by investigating the influence of Po-P on subsequent disease outcome in our cohort of women with recent onset IP. We also explored the impact of autoantibody status.

\section{PATIENTS AND METHODS}

\section{Patients}

The cohort comprised consecutive women aged $<48$ years at symptom onset (our oldest recorded age at pregnancy), who were registered with the Norfolk Arthritis Register (NOAR) between January 1990 and December 2004. NOAR is a primary care-based inception cohort of patients with recent onset IP.7 Patients underwent a standardised assessment by a research nurse at baseline and years 1,2 , $3,5,7,10$ and 15 .

\section{Data collection}

At each assessment the patient completed the British version of the Stanford Health Assessment Questionnaire (HAQ). ${ }^{8}$ Blood samples taken at baseline and during follow-up were tested for RF (latex method; positive result was a titre $\geq 1: 40$ ) and ACPA (Axis-Shield DIASTAT kit, Axis-Shield, Cambridge, UK; positive: $>5 \mathrm{U} / \mathrm{ml})$. At each assessment the patient was asked about the dates and outcome of any pregnancies. Pregnancies resulting in multiple births were counted as one live birth. Women were regarded as 'pregnant at assessment' if their assessment took place less than 40 weeks before the date of delivery.

\section{Statistical analysis}

Independent samples $t$ tests, Wilcoxon/MannWhitney tests, $\chi^{2}$ tests and logistic regression, as appropriate to the data characteristics, were used to test for differences in the baseline characteristics of women with and without a Po-P. Linear random effects (LRE) models were used to compare HAO score over time, by pregnancy status (see supplementary material). We defined pregnancy status as a non-time variable constant based on whether patients had a Po-P or not at any time during followup, and then as a time variable factor (not yet had a Po-P vs had a Po-P) which allowed for the timing of pregnancies and comparison of the HAQ scores of women who had a pregnancy, with their estimated trajectory had they not had a pregnancy. For the LRE models, patients were classified as 'positive' for RF or ACPA, if they had a positive test result at any time post onset. Adjustment was made for age online under the BMJ Journals unlocked scheme, see http:// ard.bmj.com/info/unlocked.dtl 
at symptom onset and symptom duration at baseline in all of the LRE models. All analysis was carried out using STATA V.10 (StataCorp, College Station, Texas, USA).

\section{RESULTS}

\section{Baseline characteristics}

A total of 631 women satisfied the entry criteria for the study. Their baseline characteristics are presented in table 1 . The median (IOR) follow-up time was 6.9 years $(4.1-12.2)$ and the total follow-up time was 4733 person-years. In all, 72 (11.4\%) women had at least 1 pregnancy during follow-up, and 45 women were pregnant at the time of an assessment.

Women who had a Po-P were younger at symptom onset, less likely to be positive for ACPA, or meet the American College of Rheumatology (ACR) criteria for RA, more likely to be taking the contraceptive pill at baseline, and had fewer previous pregnancies than women who did not have a Po-P (table 1). There were no significant baseline differences in disease activity between women who did and did not have a Po-P.

\section{Baseline associations with Po-P}

Younger age at symptom onset was a significant predictor of Po-P (table 1). Neither baseline HAO nor 28-joint Disease Activity Score (DAS28) results were significantly associated with Po-P.

\section{Po-P and HAO score}

The estimated changes in HAQ score by Po-P status (non-time variable) are shown in table 2 . For clarity, we have only presented results at years 1, 5, 10 and 15 of follow-up. From year 5 onwards, women with a Po-P had significantly lower HAO scores than women who did not have a Po-P.

\section{Time variable pregnancy status and $\mathrm{HAO}$ score}

When pregnancy was treated as a time variable factor, women who had a pregnancy during follow-up had lower HAO scores over time than they would have done had they not had a pregnancy by that time $(-0.16 ; 95 \% \mathrm{CI}-0.25$ to -0.06$)$, adjusting for age at symptom onset, symptom duration at baseline and number of previous live births.

Table 1 Baseline characteristics by post-onset pregnancy and baseline associations with likelihood of post-onset pregnancy (adjusted for age at symptom onset)

\begin{tabular}{|c|c|c|c|c|}
\hline Cohort characteristics at baseline & $\begin{array}{l}\text { Women who had a } \\
\text { post-onset pregnancy } \\
(\mathrm{N}=72)\end{array}$ & $\begin{array}{l}\text { Women who did not have } \\
\text { a post-onset pregnancy } \\
(\mathrm{N}=559)\end{array}$ & p Value & $\begin{array}{l}\text { OR* of post-onset } \\
\text { pregnancy }(95 \% \mathrm{CI}) \\
\text { (reference) }\end{array}$ \\
\hline Age at IP onset, years: median (IOR) & $27.8(23.1-30.4) ; n=72$ & $39.0(33.1-44.0) ; n=559$ & $<0.001 \dagger$ & $0.83(0.80$ to 0.87$) \ddagger$ (per year increase) \\
\hline Number of live births by baseline: $\mathrm{n} / \mathrm{N}(\%)$ & & & $<0.001 \dagger$ & 0.79 (0.59 to 1.06$)$ (per live birth) \\
\hline 0 & $33 / 72(45.8)$ & $106 / 559(19.0)$ & & \\
\hline 1 & $23 / 72(31.9)$ & $84 / 559(15.0)$ & & \\
\hline 2 & $13 / 72(18.1)$ & $229 / 559(41.0)$ & & \\
\hline 3 & 1/72 (1.4) & $103 / 559(18.4)$ & & \\
\hline 4 & $2 / 72(2.8)$ & 28/559 (5.0) & & \\
\hline 5 & - & $9 / 559(1.6)$ & & \\
\hline Taking contraceptive pill at baseline: $\mathrm{n} / \mathrm{N}(\%)$ & $32 / 72(44.4)$ & $102 / 559(18.3)$ & $<0.001 \dagger$ & 1.58 (0.88 to 2.85 ) (vs not taking) \\
\hline Symptom duration, months: median (IQR) & $7.5(3.5-12.1) ; n=72$ & $6.9(3.2-14.8) ; n=559$ & 0.94 & $0.99(0.97$ to 1.00$)$ (per month increase) \\
\hline Met ACR (1987) criteria for $\mathrm{RA}^{16}: \mathrm{n} / \mathrm{N}(\%)$ & $17 / 72(23.6)$ & $199 / 559(35.6)$ & $0.04 \dagger$ & 0.59 (0.31 to 1.13$)$ (vs did not meet) \\
\hline Positive for RF: n/N (\%) & $12 / 61(19.7)$ & $122 / 486(25.1)$ & 0.35 & 1.08 (0.51 to 2.29 ) (vs negative for RF) \\
\hline Ever positive for RF during follow-up: $\mathrm{n} / \mathrm{N}(\%)$ & $19 / 70(27)$ & $215 / 539(40)$ & - & - \\
\hline Positive for ACPA: n (\%) & 6/56 (10.7) & $118 / 463(25.5)$ & $0.01 \dagger$ & 0.41 (0.16 to 1.07$)$ (vs negative for ACPA) \\
\hline Ever positive for ACPA during follow-up: n/N (\%) & $10 / 64(16)$ & $135 / 507(27)$ & - & - \\
\hline CRP concentration, mg/litre: mean (SD) & $10.0(17.8) ; n=55$ & $10.8(21.4) ; n=447$ & 0.80 & 0.99 (0.98 to 1.01$)$ (per $\mathrm{mg} /$ litre increase) \\
\hline $\begin{array}{l}\text { Swollen and tender joint count ( } 51 \text { joints): median } \\
\text { (IQR) }\end{array}$ & $1(0-4) ; n=72$ & $2(0-6) ; n=559$ & 0.36 & 0.98 (0.93 to 1.03 ) (per joint increase) \\
\hline DAS28: mean (SD) & $3.5(1.3) ; n=55$ & $3.6(1.3) ; n=447$ & 0.60 & $0.96(0.75$ to 1.22$)$ (per increase of 1.0$)$ \\
\hline HAO score: median (IQR) & $0.63(0.19-1.0) ; n=72$ & $0.75(0.25-1.38) ; n=551$ & 0.10 & $0.66(0.43$ to 1.01$)$ (per increase of 1.0$)$ \\
\hline
\end{tabular}

${ }^{*} \mathrm{OR}$, association with likelihood of having a post-onset pregnancy, compared to (reference).

†Significant difference (unadjusted).

¥Significant association with likelihood of post-onset pregnancy.

ACPA, anti-cyclic citrullinated peptide antibody; ACR, American College of Rheumatology; CRP, C reactive protein; DAS28, 28-joint Disease Activity Score; HAO, Health Assessment Questionnaire; RA, rheumatoid arthritis; RF, rheumatoid factor.

Table 2 Change in HAO score from baseline at years 1, 5, 10 and 15 of follow-up by post-onset pregnancy status, and difference between women with and without a post-onset pregnancy, adjusted for age at symptom onset and symptom duration at baseline

\begin{tabular}{|c|c|c|c|c|c|}
\hline \multirow[b]{2}{*}{ Follow-up anniversary } & \multicolumn{2}{|c|}{ Post-onset pregnancy } & \multicolumn{2}{|c|}{ No post-onset pregnancy } & \multirow{2}{*}{$\begin{array}{l}\text { Difference: pregnancy } \\
\text { versus no pregnancy } \\
(95 \% \mathrm{Cl})\end{array}$} \\
\hline & $\mathbf{n}$ & $\begin{array}{l}\text { Change in } \mathrm{HAO} \text { score } \\
\text { from baseline }(95 \% \mathrm{CI})\end{array}$ & n & $\begin{array}{l}\text { Change in } \mathrm{HAO} \text { score from } \\
\text { baseline }(95 \% \mathrm{CI})\end{array}$ & \\
\hline 1 & 70 & $-0.25(-0.44 \text { to }-0.05)^{*}$ & 542 & $-0.08(-0.13 \text { to }-0.03)^{*}$ & $-0.17(-0.36$ to 0.03$)$ \\
\hline 5 & 67 & $-0.23(-0.43 \text { to }-0.03)^{*}$ & 441 & $-0.01(-0.06$ to 0.05$)$ & $-0.22(-0.42$ to -0.02$) \dagger$ \\
\hline 10 & 27 & $-0.16(-0.40$ to 0.07$)$ & 179 & $0.10(0.03 \text { to } 0.18)^{*}$ & $-0.27(-0.51$ to -0.03$) \dagger$ \\
\hline 15 & 22 & $-0.11(-0.35$ to 0.14$)$ & 114 & $0.21(0.12 \text { to } 0.29)^{*}$ & $-0.31(-0.57$ to -0.06$) \dagger$ \\
\hline
\end{tabular}

*Significant change in $\mathrm{HAO}$ score from baseline.

tSignificant difference in change in $\mathrm{HAO}$ score from baseline, by post-onset pregnancy.

$\mathrm{HAQ}$, Health Assessment Questionnaire. 
Table 3 Influence of post-onset pregnancy status and cumulative autoantibody status on change in HAO score over time, adjusted for age at symptom onset, symptom duration at baseline and number of previous live births

\begin{tabular}{|c|c|c|c|c|}
\hline & \multicolumn{2}{|l|}{ RF status } & \multicolumn{2}{|l|}{ ACPA status } \\
\hline & Negative & Positive & Negative & Positive \\
\hline \multicolumn{5}{|l|}{ Post-onset pregnancy } \\
\hline Yes & -0.23 & 0.05 & -0.28 & 0.54 \\
\hline \multirow{2}{*}{$\begin{array}{l}\text { Difference in change in } \mathrm{HAO} \text { score by post-onset } \\
\text { pregnancy status }(95 \% \mathrm{Cl})\end{array}$} & $-0.23(-0.34 \text { to }-0.12)^{*}$ & $-0.05(-0.22$ to 0.11$)$ & $-0.28(-0.39 \text { to }-0.18)^{*}$ & $0.37(0.14 \text { to } 0.59)^{*}$ \\
\hline & Results from stratified LRE models & & & \\
\hline
\end{tabular}

In the top half of the table, autoantibody-negative women without a post-onset pregnancy are the reference group (Ref). Values represent difference in HAO score from the reference group.

* Significant difference between $\mathrm{HAO}$ scores of women who had a pregnancy and women who had not.

ACPA, anti-cyclic citrullinated peptide antibody; HAQ, Health Assessment Questionnaire; LRE, linear random effect; RF, rheumatoid factor.

\section{Pregnancy at assessment and $\mathrm{HAQ}$ score}

Women who were pregnant at assessment had significantly lower $\mathrm{HAQ}$ scores over time $(-0.18 ; 95 \% \mathrm{CI}-0.33$ to -0.03$)$ than estimated had they not been pregnant, adjusting for age at symptom onset, symptom duration at baseline and number of previous live births.

\section{Change in HAQ score and Po-P by autoantibody status}

RF-negative and ACPA-negative women who had a Po-P had significantly lower HAQ scores over time than RF-negative and ACPA-negative women who had no Po-Ps. The 10 ACPApositive women who had a Po-P had significantly higher $\mathrm{HAQ}$ scores than ACPA-positive women who had no Po-P (table 3) (see supplementary material for the HAO scores for these women). We repeated the original LRE model, with time variable pregnancy status, for each autoantibody subgroup (table 3). Similar results were found using both statistical techniques.

\section{DISCUSSION}

In our investigation we found that women who had at least one Po-P had lower HAQ scores over the entire follow-up period and following pregnancy than women who did not have a Po-P. These results do not support previous studies of long-term disease outcome following pregnancy; ${ }^{6}$ however there are many methodological differences. The relationship between Po-P status and HAQ score varied with RF and ACPA status. Confirming the findings of previous studies, ${ }^{1-39}$ we found that women who were pregnant at the time of an assessment had significantly lower estimated HAQ scores than they would have done had they not been pregnant.

Women who had a Po-P were younger at symptom onset and less likely to be ACPA positive or meet the ACR criteria for RA than those who had no pregnancy. This may be because patients with more severe disease are less likely to want to increase the size of their family. Thus the women who had a pregnancy may have had better disease outcome even if they had had no further pregnancies. However, once adjusted for age at symptom onset there were no significant differences between the two groups. The difference in $\mathrm{HAO}$ scores were non-significant until the fifth anniversary, by which time $90 \%$ of the 'had Po-P' group had been pregnant. This suggests that the two groups of women were not different before pregnancy. In either case, we have shown that, in general, pregnancy was not associated with a detrimental effect on disease outcome.

Our results support previous findings that patients with seronegative RA are more likely to experience amelioration during pregnancy than patients who are seropositive. ${ }^{4}$ Among the ACPA-positive women in our cohort, pregnancy was associated with higher $\mathrm{HAQ}$ scores than no pregnancy, although numbers are small. It is possible that pregnancy and subsequent childcare placed an added strain on those with an underlying poor prognosis ${ }^{10}$ leading ultimately to a worse outcome. Further studies in ACPA-positive women are needed to determine the influence of Po-P in this subgroup.

This is the largest prospective study to investigate the impact of Po-P in a cohort of patients with recent onset IP. The strengths and weaknesses of the recruitment of patients with IP from primary care in NOAR have been discussed elsewhere. ${ }^{7}$ One weakness of the current study is that we have not considered treatment effects. Many women stop taking treatment while they are pregnant, breast feeding or trying to conceive ${ }^{9}$ 11 which, if anything, would to lead to a worse outcome, hence more intensive treatment is unlikely to explain our findings.

The 'changing maternal 'self' hypothesis' describes how, during pregnancy, fetal cells in the mother's blood stream (microchimerism) cause the mother's immune system to reidentify 'self', resulting in an amelioration of RA symptoms. Microchimerism can persist for decades postpartum, thus resulting in a long-term improvement in disease. ${ }^{12}$ The level of fetal DNA in the mother's blood has been found to correlate with disease activity. ${ }^{13}$ However, a recent study found that microchimerism of RA-associated human leucocyte antigen (HLA) alleles is found more frequently in women with RA than in non-RA controls. ${ }^{14}$ Microchimerism may have either a beneficial (by contributing to tissue repair) or adverse (via fetal cells acting as effector cells or the targets of immune attack) effect on disease severity among patients with RA. ${ }^{15}$ It is possible that in the presence of ACPA, fetal cells have the opposite effect than in situations when ACPA is absent, or at low levels. Alternatively, the temporary amelioration of rheumatological symptoms during pregnancy may lead to a reduction in cumulative disease burden, thus producing the effect seen here.

Thus, in general, patients with IP who become pregnant after symptom onset have less functional disability than patients with IP who do not become pregnant after symptom onset. Seronegative women with IP should not be discouraged from becoming pregnant. 
Acknowledgements The support of clinical staff at the Norfolk and Norwich University Hospital and the local primary care doctors is gratefully acknowledged. Data management by Stephanie Manning and her team is also appreciated.

Funding NOAR is funded by Arthritis Research UK (Grant reference 17552).

Ethics approval This study was conducted with the approval of the Norfolk and Norwich University Hospital Local Research Ethics Committee.

Provenance and peer review Not commissioned; externally peer reviewed.

\section{REFERENCES}

1. Hench PS. The ameliorating effect of pregnancy on chronic atrophic (infectious rheumatoid) arthritis, fibrositis and intermittent hydrarthritis. Mayo Clin Proc 1935:13:161-7.

2. Nelson JL, Ostensen M. Pregnancy and rheumatoid arthritis. Rheum Dis Clin North Am 1997:23:195-212.

3. Ostensen $\mathbf{M}$, Villiger PM. The remission of rheumatoid arthritis during pregnancy. Semin Immunopathol 2007;29:185-91.

4. de Man YA, Bakker-Jonges LE, Goorbergh CM, et al. Women with rheumatoid arthritis negative for anti-cyclic citrullinated peptide and rheumatoid factor are more likely to improve during pregnancy, whereas in autoantibody-positive women autoantibody levels are not influenced by pregnancy. Ann Rheum Dis 2010;69:420-3.

5. Oka M, Vainio U. Effect of pregnancy on the prognosis and serology of rheumatoid arthritis. Acta Rheumatol Scand 1966;12:47-52.

6. Drossaers-Bakker KW, Zwinderman AH, van Zeben D, et al. Pregnancy and oral contraceptive use do not significantly influence outcome in long term rheumatoid arthritis. Ann Rheum Dis 2002;61:405-8.
7. Symmons DP, Barrett EM, Bankhead CR, et al. The incidence of rheumatoid arthritis in the United Kingdom: results from the Norfolk Arthritis Register. $\mathrm{Br} J$ Rheumatol 1994:33:735-9

8. Kirwan JR, Reeback JS. Stanford Health Assessment Questionnaire modified to assess disability in British patients with rheumatoid arthritis. Br J Rheumatol 1986;25:206-9.

9. de Man YA, Dolhain RJ, van de Geijn FE, et al. Disease activity of rheumatoid arthritis during pregnancy: results from a nationwide prospective study. Arthritis Rheum 2008:59:1241-8.

10. Kroot EJ, de Jong BA, van Leeuwen MA, et al. The prognostic value of anti-cyclic citrullinated peptide antibody in patients with recent-onset rheumatoid arthritis. Arthritis Rheum 2000;43:1831-5.

11. Barrett $\mathbf{J H}$, Brennan $\mathrm{P}$, Fiddler $\mathrm{M}$, et al. Does rheumatoid arthritis remit during pregnancy and relapse postpartum? Results from a nationwide study in the United Kingdom performed prospectively from late pregnancy. Arthritis Rheum 1999; 42:1219-27.

12. Adams Waldorf KM, Nelson JL. Autoimmune disease during pregnancy and the microchimerism legacy of pregnancy. Immunol Invest 2008;37:631-44.

13. Yan Z, Lambert NC, Ostensen M, et al. Prospective study of fetal DNA in serum and disease activity during pregnancy in women with inflammatory arthritis. Arthritis Rheum 2006;54:2069-73.

14. Rak JM, Maestroni L, Balandraud N, et al. Transfer of the shared epitope through microchimerism in women with rheumatoid arthritis. Arthritis Rheum 2009;60:73-80.

15. Nelson JL. Naturally acquired microchimerism: for better or for worse. Arthritis Rheum 2009;60:5-7.

16. Arnett FC, Edworthy SM, Bloch DA, et al. The American Rheumatism Association 1987 revised criteria for the classification of rheumatoid arthritis. Arthritis Rheum 1988:31:315-24. 